\title{
RECURSOS HÍDRICOS ALTOANDINOS, ESTRATEGIAS DE DESARROLLO ECONÓMICO Y PROYECTOS DE RIEGO: TARAPACÁ, 1880-1930
}

\author{
HYDRAULIC RESOURCES OF THE HIGH ANDES, STRATEGIES \\ OF ECONOMIC DEVELOPMENT AND IRRIGATION PROJECTS: \\ TARAPACÁ, 1880-1930 \\ Luis Castro C.*
}

\begin{abstract}
Este artículo aborda la problemática referida al uso de los recursos hídricos del área andina de la provincia de Tarapacá entre las décadas de 1880 y 1930 contextualizada en el debate epocal sobre el desarrollo regional. Describe las distintas iniciativas que se plantearon y los fundamentos propositivos que las sustentaron. Además, analiza la visión del Estado chileno respecto a la utilización de los recursos naturales del espacio altoandino tarapaqueño, las posiciones y argumentaciones de la comunidad provincial en torno a este tema, y el impacto potencial que pudieron tener estos proyectos de uso de acuíferos cordilleranos en las comunidades aymaras locales.
\end{abstract}

Palabras claves: Recursos hídricos altoandinos, estrategia de desarrollo regional, irrigación, recursos Aymara.

This article concerns water usage and regional development in the Andean province of Tarapacá between the decades of 1880 and 1930. Various initiatives during this period were proposed and considered. Presented here is the vision of the Chilean state regarding the utilization of natural resources of the Tarapaca highlands, the positions and arguments of the community, and the potential impact that these projects had on mountain aquifers for Aymara communities.

Key words: Andean water resources, regional development, irrigation, and Aymara resources.

En el contexto del debate público sobre la problemática del desarrollo regional llevado a cabo en la provincia de Tarapacá (Figura 1) durante las primeras seis décadas de administración chilena, uno de los tópicos que provocó mayor preocupación fue el de los recursos hídricos (Castro 1995:32-66, 1998a:390-433).

Desde el punto de vista del Estado chileno el tema pasaba exclusivamente por asegurar una dotación suficiente de agua para mantener la base industrial y social que permitiera la producción de nitratos. Cualquier otro uso debía supeditarse a este propósito fundamental. Para los intereses de los actores locales involucrados en el debate el asunto tuvo ribetes contradictorios. Algunos promovieron la expansión agrícola mediante una mayor y mejor utilización de los acuíferos. Otros, en cambio, exigieron a la administración estatal la ocupación de enormes cantidades de recursos acuícolas con fines netamente de modernización: potabilizada para consumo doméstico, riego ornamental y uso industrial urbano (Castro 1998a:433-454).
A diferencia de lo planteado por Juan van Kessel (1985:142-145, 1992:196) de que las acciones por expandir el sector agrario durante el ciclo expansivo del salitre obedecieron únicamente a la mirada cortoplacista de empresarios y autoridades chilenas destinada a satisfacer las demandas emanadas de los centros productivos del nitrato de sodio y que estos ímpetus se diluyeron rápidamente por el alto costo de la infraestructura requerida y por lo limitado del mercado consumidor potencial, postulo que el uso de los acuíferos bajo un parámetro modernizador y estratégico fue un punto de desencuentro entre dos perspectivas económicas. Una discordia que adicionalmente hizo abortar la opción de algunos tarapaqueños por lograr un crecimiento agrícola local sustentado en un modelo que optimizara la acumulación hídrica para el riego.

En este sentido más que concordancia coyuntural entre Estado y empresarios salitreros para incentivar cierta actividad agrícola destinada al consumo de quienes trabajaban en las faenas

* Facultad de Humanidades, Universidad de Valparaíso, 2 Norte 802, Viña del Mar. Becario Mecesup, Programa Doctorado en
Historia, Universidad de Chile.luis.castro@uv.cl; quismalc@ yahoo.com Recibido: abril 2001. Aceptado: marzo 2004. 


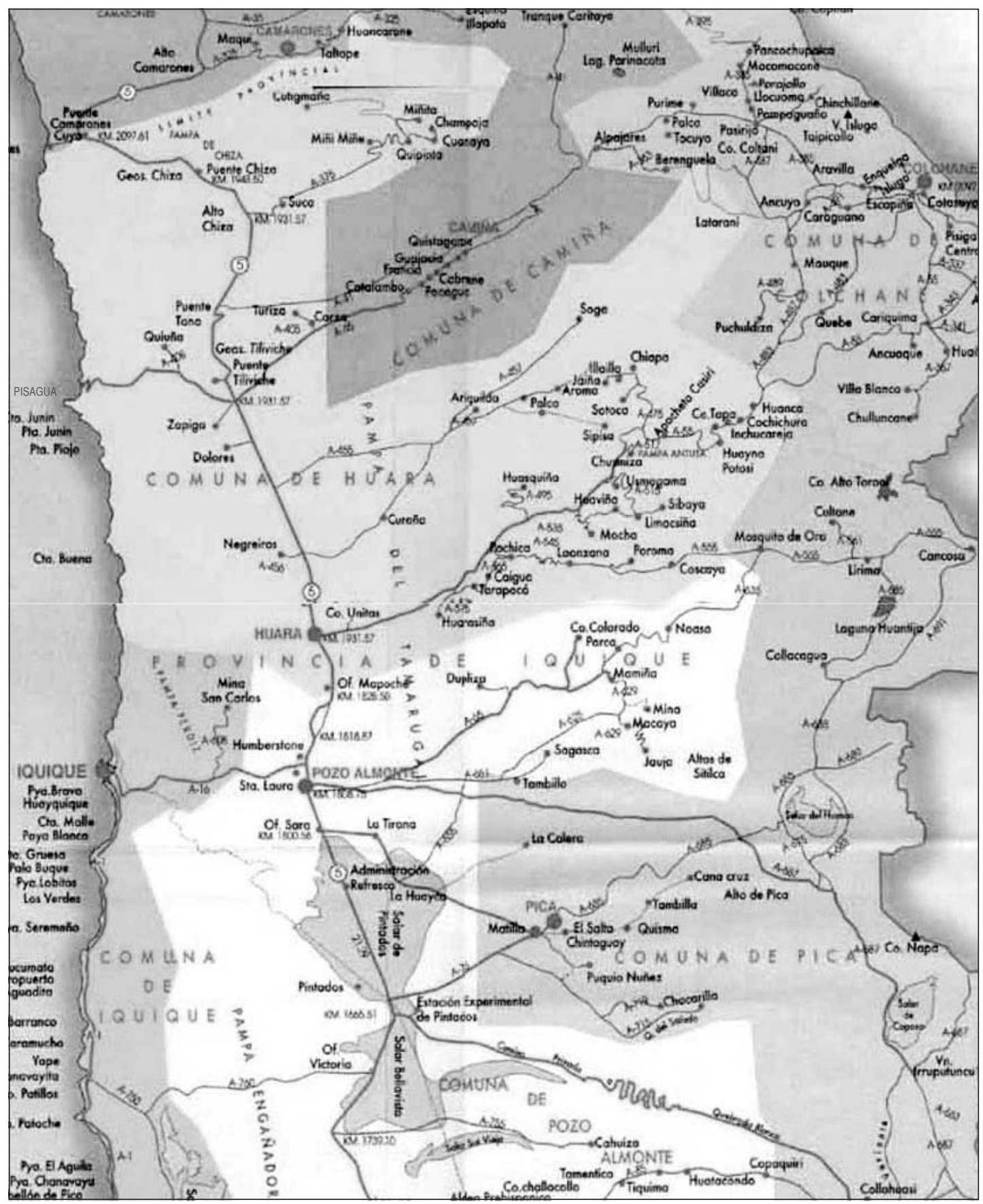

Figura 1. Mapa de la Provincia de Iquique (antiguamente Provincia de Tarapacá).

Map of the Iquique province, former Tarapacá province.

salitreras, lo que hubo fue un proceso conflictivo entre el empecinamiento estatal por asegurar la dotación de recursos que resguardara la producción de salitre, y así cobrar la anhelada renta derivada por cada quintal exportado de este fertilizante salino, y el interés decidido de activos actores políticos, gremiales y del comercio iquiqueño por habilitar un sector agrícola de magnitud en la pampa del Tamarugal al amparo de un modelo de desarrollo regional no rentista, es decir, no centrado en la explotación del llamado "oro blanco" y la carga tributaria asociada. 
Si bien el debate público derivado a partir de esta oposición de intereses y visiones económicas consignó una genuina preocupación en importantes agentes locales por resguardar los recursos hídricos para la agricultura, del mismo modo acentuó una marginación estructural de los campesinos indígenas, tributarios históricos de este bien natural, en el ámbito de los contenidos planteados a nivel de los proyectos de riego a gran escala como en la proyectada articulación social de los beneficios que se anhelaban. Esta deficiencia contenida en las distintas propuestas locales de trasvasije de aguas altoandinas a la pampa del Tamarugal no sólo se verificó en la total despreocupación por el potencial impacto negativo que podría producir esta acción en las economías agroganaderas de las poblaciones indígenas de la provincia al correr el riesgo de quedar literalmente sin recursos hídricos, sino también en la escasa injerencia que tuvo en los años 1912 a 1924, en tanto una posición pro utilización agrícola de las aguas, en la generación de una opinión pública contraria al uso de los acuíferos de las vertientes de Chintaguay ubicadas en el valle de Quisma con el objeto de abastecer a Iquique (Castro 1998a).

En resumen, el siguiente trabajo aborda la problemática pública que suscitó el manejo de las aguas altoandinas con fines económicos entre las décadas de 1880 y 1930 y los efectos que pudieron generar algunos de los planteamientos regionalistas partidarios del traslado de los recursos hídricos cordilleranos a la pampa del Tamarugal en las economías agroganaderas de las poblaciones indígenas aymara parlantes de Tarapacá. Con este fin se describirán los distintos proyectos que buscaron irrigar los suelos pampinos de la provincia, y se revisarán algunos contenidos explicitados al interior de la idea de desarrollo regional y expansión agrícola.

\section{Una larga Historia: La Utilización de los Recursos Hídricos Altoandinos para la Irrigación del Desierto}

En Tarapacá las intenciones por darle un sentido estratégico al uso de las aguas, quizás por sus características geoclimáticas, fue una constante desde el período precolombino. Ya en la época del dominio incaico se había intentado canalizar a gran distancia y volumen acuíferos cordilleranos para regar la pampa del Tamarugal (Hidalgo 1985:183; van Kessel 1985:145; Guerrero 1991:6). Durante el lapso colonial dos iniciativas continuaron con esta idea: la de O'Brien, que en 1765 buscó regar el sector norte de esta planicie conocida como pampa Iluga, y la de Mendizábal, que en 1807 planteó encauzar los recursos hídricos de la quebrada de Tarapacá con este mismo objetivo (Hidalgo 1985:186-192; Larraín 1974:9-12; Couyoumdjian y Larraín 1975:329-362).

Bajo la administración peruana los esfuerzos por irrigar el área ecológica de la depresión intermedia de Tarapacá se acrecentaron significativamente, sobre todo por la configuración de un mercado consumidor creciente al amparo de la industria salitrera. En 1845 el ingeniero chileno Francisco Puelma fue el primero en sugerir, en este nuevo y capitalista entorno epocal, la viabilidad del proyecto para cultivar el llano del Tamarugal, una idea que la retomó en 1857 el ingeniero Smith (Hidalgo 1985:186-192; Larraín 1974:9-12; Couyoumdjian y Larraín 1975:329-362). En 1863, comisionado por el gobierno del Perú, el ingeniero británico Church propuso regar esta pampa y las tierras viñateras del oasis de Pica con aguas cordilleranas de la laguna del Huasco (Hidalgo 1985:186-192; Larraín 1974:9-12; Couyoumdjian y Larraín 1975:329-362). En 1872, Oton Buchwald practicó un reconocimiento de factibilidad agrícola que se tradujo en un informe emitido el 13 de mayo de 1873. En este estudio se sostuvo la necesidad de repoblar el bosque de tamarugos que se había talado indiscriminadamente para utilizarlo como combustible en el proceso de obtención del nitrato de sodio bajo el sistema de paradas (Billinghurst 1887:152-153; Larraín 1974:9-12) ${ }^{1}$. En este mismo año el científico italiano Antonio Raimondi vuelve a insistir en la viabilidad técnica del riego de la pampa del Tamarugal con el objeto de desarrollar una agricultura de grandes dimensiones (Billinghurst 1886:105; van Kessel 1992:196; Bermúdez s/f:415)². Por último, en 1876 agricultores del valle de Tarapacá, como Antonio Cevallos, Fermín Vernal, Félix Olcay, Eduardo Caucoto, Manuel Vernal, Marcelino Luza, Andrés Loayza, Mariano Perea y otros, crean una sociedad anónima destinada a implementar un proyecto de irrigación de la quebrada y planicie aledaña desaguando aguas cordilleranas ${ }^{3}$.

Hacia la década de 1880, a pesar del cambio de administración política de la provincia a manos chilenas, este proceso no se detuvo; aún más, co- 
menzó a teñirse de cierto sesgo de modernización. Por ejemplo, a mediados de 1884 Claudio Mackenna pidió autorización para ocupar acuíferos de los ríos Loa y Collacagua y de las lagunas de Lirima, Coposa y Huasco, con el propósito de hacerlos llegar a los "terrenos fiscales [de la pampa] susceptibles de regarse a gran escala"4, una idea que llevó a la máxima autoridad política de la provincia a señalarle al Ministro de Obras Públicas que:

La idea en sí misma no puede menos que merecer el apoyo del gobierno.

Si llegara a realizarse convertirá los campos feraces, susceptibles de una producción intertropical en un desierto que hoy día no tiene en sí mismo valor alguno, y que más bien son por su esterilidad y abandono [es] un obstáculo o un peligro para todo hombre que se aventura en ellos.

Aunque dudo que las ideas que predominan en esta solicitud lleguen jamás a realizarse, es conveniente que por parte de la autoridad no encuentren sino facilidades y estímulo.

La creación de la agricultura en la pampa del Tamarugal sería lo mismo que la improvisación de una fuente de riqueza que superaría tal vez a la del salitre ${ }^{5}$.

En 1885, continuando con esta dinámica, Francisco S. Olivan solicitó una concesión de aguas subterráneas de la zona cordillerana con el objeto de irrigar la pampa del Tamarugal ${ }^{6}$. Muy entusiasmado con esta solicitud, el Intendente Gonzalo Bulnes le hizo llegar al Ministro del Interior ideas muy similares a las dirigidas al ministro de Obras Públicas un año antes a propósito del interés de Mackenna de acometer este mismo proyecto:

El Sr. Olivan se propone utilizar por medio de conocimientos exitosos y eventuales, esas aguas subterráneas extrayendo del fondo de la tierra una riqueza ignorada. Este sólo [hecho] basta para que su empresa merezca la decidida protección del Gobierno. Si a esto se añade que un ensayo feliz tentaría otras empresas análogas y que al favor de estas tentativas podrían formarse oasis regados en medio de la pampa, US. comprenderá sin esfuerzo que inmensas ventajas proporcionaría esta nue- va industria a la vida y al bienestar de los habitantes de la provincia ${ }^{7}$.

Las solicitudes para buscar y utilizar recursos hídricos altoandinos, dado el respaldo de la máxima autoridad política de Tarapacá, aumentaron notablemente. A mediados de 1886 Olivan pidió nuevas concesiones ${ }^{8}$. Por esa misma fecha Temístocles Larrañaga, Juan Nepuceno Mutis y Samuel Villalobos demandaron mercedes de agua ubicadas al interior de Pica para incrementar trabajos de cultivos mayores en este oasis ${ }^{9}$. En 1887 los empresarios franceses Le Chevalier Fontaine y Eduardo de Lapeyrousse elevaron una petición en este sentido para experimentar agrícolamente la pampa salitrera ${ }^{10}$, y en el valle de Camarones se dio forma a una sociedad agrícola con el propósito de plantar intensivamente alfalfa, un negocio que se pensaba solventar mediante la irrigación masiva y una comercialización a través de medios modernos de transporte como buques a vapor ${ }^{11}$. A comienzos de 1889, Nemesio Cancho gestionó una autorización para buscar aguas subterráneas destinadas a fertilizar la planicie del Tamarugal ${ }^{12}$. Ese mismo año Máximo Urízar, Eleuterio Dañin y Manuel Vidaurre del Río tramitaron una concesión a perpetuidad de todas las aguas de la laguna del Huasco al interior de Pica. El objetivo de estos empresarios fue canalizar los acuíferos hacia la pampa, acumularlos e irrigar extensivamente ${ }^{13}$. En el mes de febrero de 1890 el Intendente Ramón Yávar negó el carácter perpetuo de ésta, autorizando sólo el uso por dos años con fines exclusivamente agrícolas ${ }^{14}$. A la par con esta negativa de la máxima autoridad política de la provincia, el gobierno decretó el 22 de enero de 1890 el traslado a esta región del químico Juan Shulze, de la Universidad de Chile, con la misión de realizar reconocimientos destinados a ver la factibilidad de conducir aguas de las lagunas cordilleranas emplazadas al interior de la quebrada de Tarapacá hacia la pampa del Tamarugal e Iquique ${ }^{15}$.

En este escenario, el ingeniero Valentín Martínez publicó en 1893 el primer informe metódico del sistema hídrico de Tarapacá, un texto en donde se dejó claramente establecido, a pesar de algunos errores, la mayor potencialidad de las existencias acuícolas altoandinas:

De las cinco zonas en que puede dividirse la provincia de Tarapacá, la de la costa y 
la de los caliches no ofrecen ningún recurso en agua potable. La zona de la pampa del Tamarugal podría ofrecer recursos si existiesen capas subterráneas artesianas; pero todos los ensayos hechos hasta hoy manifiestan que el terreno de la pampa es un terreno de acarreo impregnado de agua, pero sin presión, como que tiene su origen en el agua de la zona de las quebradas que alcanza a llegar a la pampa por escurrimiento superficial en los fuertes aluviones.

La zona de las quebradas, en las cuales comprenderemos el oasis de Pica y la altiplanicie andina, son las únicas que ofrecen recursos en agua potable. Ahí hay lagos, ríos y vertientes; pero, por encontrarse el terreno impregnado de sales tanto las aguas de las lagunas como la de los ríos son salobres. Quedan sólo las vertientes, las cuales es de absoluta necesidad captarlas en su origen, por encontrarse impregnados de sales los terrenos por donde corren (Martínez, V. 1893:6).

Sobre los alcances de su estudio, Martínez propuso a fines de 1894 utilizar las aguas de las vertientes de Yabricoya con el doble objeto de irrigar la pampa y abastecer a la población iquiqueña ${ }^{16}$.

En la década de 1900 el interés por ocupar acuíferos altoandinos no decayó. En 1903 se le entregaron en privilegio a Manuel Risueño las aguadas de Anchoquinquintipa, Pancheta, Milagro y Petentecaya ubicadas en Guatacondo ${ }^{17}$. En 1906 las aguas de Piga fueron concesionadas a Raimundo Salas para regadío y usos industriales ${ }^{18}$. Sobre la base de este permiso, el ingeniero Camilo Edwards estudió la posibilidad de canalizar y entubar estas existencias acuícolas hacia la pampa del Tamarugal e Iquique por encargo de la sociedad comercial Compañía Proveedora de Agua de Tarapacá ${ }^{19}$. Durante 1908 se otorgaron, por un lapso de nueve años, a Carlos Aguirre Naranjo las vertientes de aguas termales y algunas vegas de Lirima ${ }^{20}$; adicionalmente los recursos hídricos de las lagunas de Lirima y Chuncara y de los riachuelos Sitana, Rinconada, Toruna y Collacagua fueron solicitados por Ramón del Barrio González ${ }^{21}$, gestión que encontró un decidido respaldo de destacados residentes iquiqueños, quienes emitieron un manifiesto público, avalado por 375 suscriptores $^{22}$, para expresar los fundamentos de su posición favorable a este proyecto:

Por su abundancia no sólo será suficiente para abastecer a la ciudad sino también a la pampa salitrera y a todos los establecimientos anexos a la explotación del salitre. Y todavía habrá de sobrante que se pueda destinar al incremento de la agricultura de la provincia. Los estudios practicados demuestran a este respecto que hay grandes extensiones de terrenos aptos para el cultivo agrícola y que sólo requiren el abono del agua que sean un elemento de riqueza para esta provincia ${ }^{23}$.

En 1911, la empresa Mannesman realizó nuevos estudios para evaluar opciones técnicamente factibles para el traslado de líquido con fines comerciales diversos ${ }^{24}$. En el mes de febrero de 1912 se decretó la expropiación de las aguas de las vertientes de Chintaguay, ubicadas en el valle de Quisma, con el propósito de llevar a cabo un proyecto fiscal de abastecimiento para Iquique (Castro 1998a:214-228, 1998b:48-54). La ejecución de esta iniciativa alcanzó ribetes insospechados, llegando a provocar un intenso conflicto que llegó a las puertas del mismo palacio presidencial y el hemiciclo parlamentario. En 1914 el ingeniero a cargo de las obras fiscales de la aducción hacia el principal puerto de la provincia, Enrique Döll, propuso utilizar las aguas de Piga y Porqueza tanto para regar el bosque de tamarugos como para abastecer a la población iquiqueña, una idea que fue finalmente rechazada por entenderse de un alto $\operatorname{costo}^{25}$.

A comienzos de 1919 Johannes Felsch planteó ocupar los acuíferos de Mamiña y las vertientes de Culumtucsa y Picunticsa para bajarlos a la zona de la pampa aledaña al pueblo de Pozo Almonte, y los recursos de las aguadas de Chara y Alona para llevarlos al oasis de Pica con el propósito de irrigar y potenciar la agricultura del lugar, disminuyendo así los efectos de la expropiación de las existencias acuícolas de las vertientes de Chintaguay (Felsch 1920:30-34; Castro 1998a:272$275)^{26}$. En el mes de mayo de 1922 el empresario Julio Pinto pidió importantes mercedes de agua de los ríos Ajutamas y Caritaya, ambos cursos afluentes del río Camarones, para llevar a cabo experimentos agrícolas ${ }^{27}$. Al año siguiente, el 15 de diciembre de 1923, el Ministerio de Obras Públicas 
le concedió a Federico Branmüller una merced de mil quinientos litros por segundo del río del valle de Aroma ${ }^{28}$. Este beneficio contempló la construcción de un tranque a cuatrocientos metros aguas abajo de la confluencia de las quebradas de Aroma y Chismiza y el derecho del gobierno a aprovechar los sobrantes de estos recursos hídricos "para regadío u otros fines que estime conveniente sin gravámenes ni indemnizaciones de ningún género" 29 . A finales de la década de 1920 Carlos Harms formuló, marcando el epílogo de las demandas de aguas cordilleranas iniciadas a mediados del siglo XIX, el último proyecto buscando sustentar la viabilidad de la anhelada estrategia de desarrollo agrícola sin menoscabar los requerimientos de modernización urbano-industrial (Carevic 1989:10).

\section{Los Afanes por Irrigar en Tarapacá Durante el Ciclo Salitrero y su Relación con un Proyecto de Desarrollo Regional}

Cobijada en una larga experiencia de intentos por llevar a cabo una irrigación a gran escala, el tema de la expansión agrícola rápidamente se asentó en la opinión pública de los tarapaqueños bajo el marco de una discusión promovida, impensadamente, por la administración chilena en consideración de su política económica asociada a la explotación del salitre, como por el especial interés de algunos sectores de la comunidad iquiqueña por configurar el uso de los recursos hídricos como parte de una estrategia de desarrollo regional. En efecto, la estrategia rentista estatal, es decir el cobro de un impuesto específico a cada quintal de salitre que saliera por los puertos nortinos, se solventó sobre la base de dos componentes: la hegemonía minera, que restringió el potencial industrial de Tarapacá en esta área en torno a la exclusiva explotación del nitrato de sodio; y la especialización productiva, que terminó condicionando a la economía tarapaqueña a una nula diversificación productiva al definirla como un espacio estrictamente minero, y en donde "lo minero" se acotaba estrictamente en "lo salitrero". En tanto, los planteamientos locales se configuraron en torno a un proyecto de desarrollo regional que contemplaba la articulación de tres sectores estratégicos: la activación de una minería regional, aspecto que buscó extender la percepción estatal de lo salitrero hacia la amplia potencialidad que había para trabajar yacimientos metálicos y no metálicos cordi- lleranos; la integración económica, comprendida como una red vial de conexión comercial a gran escala con Bolivia, el noroeste argentino y el sur peruano; y una industria agrícola, requerida a partir de un uso planificado y masivo de los recursos hídricos para cultivar comercialmente la pampa del Tamarugal (Castro 1995, 1998a, 2002).

Ya a mediados de la década de 1880, aduciendo que la agricultura del desierto sería una cuestión de primer orden en el devenir económico de la provincia de Tarapacá, tanto el Intendente Gonzalo Bulnes como el afamado Guillermo Billinghurst señalaron la necesidad de afrontar integralmente el tema de la expansión agrícola en la pampa del Tamarugal. Bulnes expresó, apoyando la solicitud de Claudio Mackenna para ocupar gratuitamente terrenos pampinos fiscales para fines agropecuarios, que:

...el Estado debe dar cualquier extensión de la pampa del Tamarugal a quién lo pida con el objeto de darle vida con su industria o sus capitales, porque en sí no tiene valor intrínseco alguno.

El interés del Estado consiste en que se cree una riqueza donde no la hay, para deducir de allí los provechos fiscales que sólo la riqueza puede $\mathrm{dar}^{30}$.

En tanto Billinghurst pidió la entrega de incentivos a los privados para que emprendieran esta tarea mientras las instancias fiscales no fueran capaces por sí mismas de cultivar extensivamente la desértica depresión intermedia de Tarapacá:

Mientras se presenta una oportunidad favorable para que el Estado emprenda obras de esta naturaleza [acumulación y regadío] habría conveniencia en estimular en tal sentido el interés de los particulares ofreciéndoles, bajo determinadas condiciones, la propiedad de los terrenos fiscales que consigan habilitar para el cultivo (Billinghurst 1886:37).

La idea del rol estatal, para afianzar una industria agrícola en la pampa del Tamarugal, Guillermo Billinghurst la reiteró con mayor profundidad, argumentos y sentido crítico en 1893 :

No se explica uno tan pronto, cómo ese interesante llano [la pampa del Tama- 
rugal], donde se han acumulado inmensos depósitos de humus, y donde todavía se encuentran los restos palpitantes de una no remota y vigorosa vegetación, rodeado como está de desiertos áridos e inclementes, lo cual es para su cultura otro poderoso aliciente, no haya sido, de antiguo, abundantemente irrigado; y cómo los poderes públicos no han fomentado la industria privada a este respecto, ni ellos se han preocupado de una manera práctica de llevar a término esa obra que está llamada a asegurar a esta sección territorial, hasta cierto punto, una vida permanente que no se halle sujeta a las alternativas de una industria que tiene sus días contados (Billinghurst 1893:5-6).

Estas estratégicas insinuaciones de Billinghurst tuvieron la virtud de centrar la discusión relacionando algunos de los elementos medulares de la problemática por el uso del agua. Por una parte, la estabilidad agrícola versus la inestabilidad salitrera; por otra, la necesaria articulación de las iniciativas privadas con las gestiones públicas para regar el desierto. De este modo, la irrigación se comenzó a entender como un factor irrestrictamente enlazado con una propuesta de desarrollo regional. Un diseño que debía comprender en su conjunto al sector minero, al comercio interregional con Bolivia y el noroeste argentino y la actividad agrícola orientada al mercado en la pampa del Tamarugal (Castro 1995).

Este fue el sentido analítico del informe emitido en julio de 1894 por quien ocupara el cargo de Ingeniero de la Provincia:

Como la extensión cultivable de la pampa es tan considerable y existe agua en diferentes niveles y en algunos puntos casi en la superficie, debiera utilizarse abriendo pozos y estancar agua para el regadío para formar así grandes siembras de árboles frutales, potreros, huertas, etc., lo que creo fácil y muy conveniente realizar o llevar a cabo permitiendo las concesiones de terrenos para el cultivo.

De esta manera se conseguirá mayores entradas fiscales y se daría vida propia a esa extensa y desierta pampa ${ }^{31}$.
También tuvieron esta orientación los comentarios manifestados, en septiembre de 1894, por el Intendente Ruperto Álvarez al informar al Ministro de Obras Públicas su posición ante la necesidad de cultivar la superficie del Tamarugal:

El estudio de irrigación de la pampa debe, pues, iniciarse por el Supremo Gobierno con el empeño que requiere una obra de gran importancia, que reportaría incalculables beneficios al país y muy especialmente a esta provincia.

Además que le daría vida propia, la conversión de la extensa pampa hoy estéril e improductiva, en terrenos adaptables a la industria agrícola sería el medio más eficaz para dar estabilidad a las poblaciones del interior.

La irrigación de la extensa pampa del Tamarugal, sería obra que además de la importancia que en si representa, vendría a dar vida propia a la provincia de Tarapacá, que en la actualidad sólo tiene lo que le da la industria del salitre. Y si bien es cierto que por ahora no hay temor de agotamiento de la sustancia que le sirve de base, no es posible, sin embargo, prever las alternativas que puede experimentar en lo futuro ${ }^{32}$.

Las expresiones del Intendente Álvarez vinieron a remarcar, contradiciendo los intereses rentistas de la administración central, dos ideas que ya estaban circulando con fuerza en el meollo de la opinión pública tarapaqueña fines del siglo XIX. Primero, que la economía provincial no podía contener un crecimiento sostenido y estable basándose exclusivamente en la minería del salitre, y que, por tanto, era necesario potenciar otras áreas productivas más seguras en su futuro, particularmente la agricultura. Segundo, que esta materia debía ser un componente básico para una visión coherente del desarrollo regional.

Los alcances de esta opinión pionera encauzaron una creciente articulación social vinculada a la posibilidad cierta de llevar a cabo una propuesta de desarrollo regional desligada de los intereses rentistas exógenos del Estado chileno, sobre todo, porque, a partir de la posición manifestada por quien fuera entonces la máxima autoridad política de Tarapacá, se allegaron a este tipo de planteamien- 
tos algunos agentes de la administración provincial, la Municipalidad de Iquique, el periodismo ${ }^{33}$, varios gremios, colonias de residentes extranjeros, partidos políticos, la Iglesia Católica ${ }^{34}$, comerciantes y empresarios locales como Juan Dassori, Luis Junois, Bernardo Digoy, Juan Bautista Montenegro, Demófilo Herrera, Arturo del Río y Luis Advis, todos decididos emprendedores de proyectos agrícolas $^{35}$

La configuración de este soporte social conllevó un salto cualitativo que le permitió a la opinión pública iquiqueña, hacia comienzos del siglo $\mathrm{XX}$, pasar de insinuaciones y recomendaciones a demandas claramente inducidas y contestatarias. Por ejemplo, en el año 1900, tras una sostenida campaña periodística para resguardar los algarrobos existentes en los bosques pampinos, no sólo se le terminó pidiendo al fisco su directa intervención en este asunto para consolidar una relevante "posibilidad económica para la provincia" ${ }^{36}$, sino además el Delegado Fiscal de Salitreras presentó un lúcido plan para desarrollar la agricultura que contemplaba la promulgación de una ley que debía otorgar la propiedad de la tierra a quienes cultivaran el árido y salino suelo del Tamarugal (Bermúdez s/f:419). Este tipo de exigencias caló tan hondo en los vericuetos de La Moneda, que en 1903 el Ministro de Hacienda del gobierno del Presidente Germán Riesco, Manuel Salinas, presentó al hemiciclo parlamentario un proyecto de irrigación del área de Canchones que en su fundamentación sostenía:

Si fuera posible dotar de agua estos terrenos, se lograría asegurar la vida de la provincia de Tarapacá, que hoy se sostiene casi exclusivamente de industrias inestables y transitorias, como la explotación de sus depósitos de nitratos y de sus minas... (Salinas 1903:36).

La aceptación social de esta idea que aseguraba que la industria salitrera iba a terminar siendo nociva para la economía de Tarapacá debido a su endémica inestabilidad, definitivamente asentó aquello de que la potenciación agrícola a gran escala era uno de los instrumentos más adecuados para solventar en el largo plazo el desarrollo regional. Así lo planteó en su Memoria Anual, en el transcurso del año 1912, Manuel Urrutia en su calidad de Administrador de la Aduana y Tesorería Unida de Iquique:
La agricultura no es acá actualmente una industria, porque no existe en sí. Pero es un problema no debatido, que los extensos terrenos del Departamento se adaptan a ella y que puede resolverse con obras de irrigación para aumentar su poder productivo e incrementar, más que la misma minería, la fortuna pública y privada del país ${ }^{37}$.

También insistieron en lo mismo, aunque de modo más directo, los editoriales del semanario católico "Las Cuestiones Sociales" aparecidos en enero de 1922 y diciembre de 1926. En la primera, escuetamente se comentaba:

...que [la agricultura] es una riqueza que no está expuesta como las minas, o el salitre, a falta de utilidad, crisis, tempestades o calamidades o incertidumbre de ningún género ${ }^{38}$.

En tanto en la segunda, a propósito de la participación de los parlamentarios de la zona ante una supuesta iniciativa gubernamental para implementar proyectos de irrigación en el norte del país, se indicaba:

El señor Velasco, Ministro de Obras Públicas, tiende a que se destinen treinta y cuatro millones de pesos para irrigación en las provincias entre Coquimbo y Tarapacá. Si ello fuera así, habría llegado el caso que los representantes de esta provincia se esforzaran en conseguir una respetable cantidad para que la pampa pudiera convertirse en una región agrícola, principalmente estando amenazada la industria del salitre.

La pampa regable puede convertirse en campo feraz, que haría barata la vida en el norte. La región de Pica es arenosa, como toda la pampa, y, sin embargo, es cultivable hasta donde llega el agua, que, si fuera abundante habría campos fértiles inmensos. Cierto, que en terrenos altamente salitrosos, quizás no sería tan fácil la vegetación, pero muchas extensiones, aún en las mismas oficinas salitreras, que podrían dedicarse al cultivo.

Por otra parte, más allá de Pica, en Piga, hay abundante agua que se pierde lasti- 
mosamente, que bien encauzada, podría dirigirse a la pampa.

Tienen la palabra los representantes de Tarapacá en el parlamento, cuando se discuta la ley de irrigación ${ }^{39}$.

Lo marcadamente explícito de estos planteamientos devino como la resultante de una larga reflexión amparada en la observancia de la endeble realidad económica de Tarapacá. Una constatación que, además, actuó como gatillante para una radicalización de las argumentaciones de quienes optaron por un diseño de desarrollo regional totalmente desligado del rentismo salitrero al cual apostaba decididamente el Estado chileno. En este contexto, el esfuerzo más vital por viabilizar esta opción se centró de manera importante en implementar diversos proyectos agrícolas que promovieran el tema de la definición planificada del uso de los recursos hídricos existentes en este alejado y árido territorio.

Tres fueron los factores que ampararon un mayor consenso entre los diferentes actores tarapaqueños involucrados en esta discusión para pasar de iniciativas privadas focales a megaproyectos de irrigación. Primero, el que se haya entendido esta demanda dentro de un proceso promovido por la capacidad de gestión endógena para buscar un necesario respaldo estatal, despejando así algunas dudas referidas a ciertos énfasis más decididamente regionalistas de algunos discursos locales que se habían derivado de un profundo sentimiento de abandono fiscal, cuestión que no era aceptada en algunas esferas de la vida pública de Iquique considerando la importancia que tenía la zona para el modelo rentista, base de la riqueza y progreso económico del país (Castro 2003). Segundo, el que no se transara en la articulación de una lógica analítica que estuviera en función de una estrategia de desarrollo regional que potenciara y privilegiara el conjunto de las posibilidades productivas que contenía en sus entrañas esta provincia. Tercero, que el diagnóstico de la precariedad de la minería salitrera se fuera irremediablemente corroborando desde fines de la década de 1910, lo que permitió la adscripción de un número mayor de sectores sociales que se convencieron de la necesidad de superar las ideas inmediatistas y adentrarse en propuestas más estratégicas. Qué mejor ejemplo, y a la vez dramático reconocimiento a esta última postura, que la rauda desaparición de la industria salitrera en los años 1930 y 1940 . Como consecuencia de lo anterior, la provincia de Tarapacá se quedó, tras largas décadas, sólo con los fantasmas de una riqueza y un desarrollo económico prometido a su amparo; así las predicciones de inestabilidad y ausencia de desarrollo real se cumplieron con precisión hasta la aparición, necesaria y vergonzosa, de banderas y crespones negros en el otrora bullente puerto de Iquique como respuesta a una crisis terminal.

La conjunción de estos factores permitió que se le diera un respaldo social mayoritario a la propuesta de ocupar parte importante de los recursos hídricos en la implementación de un sector agrícola de grandes dimensiones en la pampa del Tamarugal, una dinámica que alcanzó incluso a aquellos que aún apostaban a la minería salitrera como el medio de crecimiento más eficaz para la economía tarapaqueña. Estos últimos tuvieron que reconocer al menos la posibilidad de complementar estas áreas productivas y, por tanto, demandar a la administración estatal mecanismos para ampliar las estructuras generadoras de riqueza al alero de un modelo suficientemente acotado y concatenado. Las resoluciones del Congreso de Cámaras de Comercio del Norte realizado en 1934 y los alcances emitidos por el estudioso Carlos Alfaro Calderón, en su "Reseña Histórica de la Provincia de Tarapacá" publicada en 1936, son una buena muestra de este aglutinamiento. Los comerciantes, en un momento socioeconómico muy difícil, llegaron a pedir:

Como medio de propender al fomento de la agricultura regional, se active la terminación de los tranques de regadío que actualmente se construyen y avanzar en los estudios de otros embalses que se recomiendan construir en las provincias de Tarapacá y Antofagasta... ${ }^{40}$.

En tanto, Alfaro Calderón acentuará, valorando lo agrícola para la economía local, la necesidad de implementar caminos para incrementar su actividad:

Aún cuando las mayores riquezas que encierra la provincia de Tarapacá son el salitre y la minería, no debe desmerecerse tampoco la agricultura, cuyos productos son variadísimos y de excelente calidad. 
Si su desarrollo no ha sido muy intenso, se ha debido esto a la escasez de transportes, como ser buenos caminos para vehículos que puedan transportar esos productos a los centros de consumo. Sin embargo, como de esto ya se ha han preocupado las autoridades desde hace poco tiempo, lo mismo que el Gobierno, se ha podido observar mayor interés entre los agricultores del interior por aumentar la producción, y sin duda que en lo sucesivo, cuando se completen las redes camineras, ese aumento será más intenso (Alfaro 1936:301).

La culminación exitosa de esta estrategia de manejo de recursos hídricos para propósitos agrícolas debió haber sido el inicio de la construcción de los tranques de Caritaya y Pachica ubicados respectivamente al interior del valle de Camarones y el poblado de Huara en la década de 1930. Sin embargo, los resabios de una administración estatal sin visión de futuro, la inconsistencia por parte de la comunidad regional a la hora de asumir un lineamiento político sin reparos en esta materia y la galopante crisis de los años 1929-1930 terminaron por dejar inconcluso este proceso de planteamientos desarrollistas en el ámbito de la agricultura en el desierto ${ }^{41}$. Ambos embalses significaron, en su momento, la posibilidad más concreta de habilitar en esta región un manejo más racional de los acuíferos, delimitando los márgenes de la discusión local respecto a la relación modernidad-agricultura. Como lo estimara el obispo de Iquique de la época, Carlos Labbé, la edificación de ambas represas:

...asegurarían a esta nuestra querida provincia un porvenir seguro y constante, basado en la agricultura que ha sido y será siempre la industria básica del progreso de nuestro país.

¡Cuán distinto será el panorama cuando se hayan terminado las obras del tranque Pachica! Ellas fecundizarán una enorme extensión de territorio, regándola con grandes canales, con agua cuyo costo, posiblemente, no excederá de la décima parte del precio del agua obtenida por medio de pozos (En Alfaro 1936:311) ${ }^{42}$.

El tranque Caritaya se ubicó a 144 kilómetros al este de Zapiga, y su objetivo primordial fue el de irrigar el valle de Camarones, un proyecto agrícola que se inició mediante el Decreto Ley promulgado el 20 de marzo de 1925 y que establecía la constitución de una Asociación de Canalistas, la especificación de los terrenos que se iban a cultivar y los mecanismos de transferencia de las mercedes de agua fiscales a los socios de la mentada asociación. Este cuerpo normativo se complementó con la aprobación, el 8 de junio de 1928, del plan confeccionado por la Dirección de Riego del Ministerio de Obras Públicas que consignaba un financiamiento de $\$ 3.300 .300$ para llevar a cabo las obras ${ }^{43}$. Se estimaba la capacidad de almacenamiento de Caritaya en unos 40 millones de metros cúbicos de agua, cantidad suficiente para fertilizar 3.000 hectáreas en Camarones y 2.000 hectáreas en las pampas aledañas. Para 1936 este depósito de acumulación se encontraba totalmente terminado (Alfaro 1936:308-309; Keller 1946:161). El de Pachica, ubicado en el valle de Tarapacá, estaba destinado a regar entre 4.500 y 5.000 hectáreas de la pampa del Tamarugal con una capacidad de depósito de 33 millones de metros cúbicos de agua (Alfaro 1936:309-310; BOIFMIT, Año II, $\mathrm{N}^{\mathrm{o}} 2$, Iquique 25/10/1936, p. 706).

Considerando el potencial de estos embalses, su mayor utilidad precisamente apuntó hacia la conformación de la trilogía base de las propuestas locales de desarrollo regional: agricultura, comercio y minería. En otras palabras, no sólo estaba en juego la posibilidad de contar con líquido suficiente para cultivar grandes extensiones de suelo seco, sino también se contaba con el hecho de que para llegar a sus instalaciones había que habilitar caminos expeditos, vías que con un poco más de impulso podían llegar a la frontera con Bolivia y de esta manera servir, por una parte, de conexión a distintas faenas mineras a ambos lados de la frontera y, por otra, a un comercio creciente entre Tarapacá y algunas ciudades bolivianas como Oruro, La Paz e incluso Cochabamba. Tanto fue así esta relación, que la propuesta del ferrocarril entre Huara y Chusmiza emprendida por el presidente del Instituto de Fomento Minero e Industrial de Tarapacá entre 1935 y 1937 se entendió como un sustento a los alcances económicos del tranque Pachica ${ }^{44}$.

Las expectativas puestas en estos proyectos fueron demasiadas. Si bien el embalse de Caritaya se terminó de construir, nunca se pudo utilizar íntegramente. Como acertadamente lo percibió Carlos Keller en la década de 1940, al ser demasiado 
grande esta obra en relación al nivel de las precipitaciones no era correcto "cifrarse expectativas exageradas en las posibilidades de incrementar apreciablemente las superficies regadas del departamento" (Keller 1946:163). Pachica, en tanto, no se concluyó. A menos de un año de haberse iniciado su habilitación las faenas se paralizaron, después continuaron, pero sin ninguna seguridad de éxito pasando a ser su silueta durante muchos años la de un gigante inconcluso ${ }^{45}$. Así lo constató el periodista iquiqueño Alberto Brandan a mediados de la década de 1930:

El tranque sigue a medio hacer, y con lo que cada año se gasta para que se invierta en la obra, no hay posibilidad que alguien garantice que en medio siglo más quede terminado (Alfaro 1936:311).

La opinión de Brandan fue coincidente con el comentario emitido en el periódico "El Regional" en diciembre de 1936:

Deplorable, bajo todos los conceptos, es la lentitud por falta de dinero, en que prosigue actualmente la construcción del tranque de Pachica, obra muy reclamada y en la cual está basado el futuro sólido de esta zona y aún de la provincia en general.

Está probado hasta la evidencia por autoridades lumbreras de la ingeniería que dicho embalse tendrá agua suficiente para su capacidad, y como si estas afirmaciones fueran pocas, la naturaleza misma con sus avenidas de agua de tiempo en tiempo, quiere demostrar a los poderes públicos de Chile que esta debiera ser una de las obras que debe merecerles su preocupación principal ya que terminada dejaría a Tarapacá una situación de seguro porvenir.

Desgraciadamente nada de esto acontece y se cree hacerlo todo con mantener en el presupuesto anual una insignificante migaja para sostener un personal inadecuado por su número y con lo cual sólo se consigue eternizar esa construcción y burlar a sí mismo las más sentidas aspiraciones de nuestra provincia ${ }^{46}$.

La frustración por estos resultados reactivó una vez más las diferencias entre la administración fis- cal y ciertos sectores organizados de Iquique más decididos en esta materia. Como nada se había logrado concretar, las estrategias de desarrollo continuaron en discusión, pero sin la fuerza de antes. Junto con la desaparición paulatina de una apuesta económica anhelada durante mucho tiempo, también se diluyó la posibilidad de reorientar la discusión referida al manejo masivo de las aguas para regadío en el contexto de una estrategia de desarrollo regional.

\section{Las Comunidades Aymaras, la Propuesta de Desarrollo Regional y los Proyectos de Irrigación con Aguas Altoandinas}

Aun cuando las propuestas locales de uso a gran escala de los acuíferos altoandinos se presentaron contextualmente como opciones menos degradantes en lo que tocaba a un uso preferentemente agrícola y no minero, sus formulaciones más sustantivas no lograron dar cuenta de todos los componentes socioculturales implicados en el asunto. La ausencia de quienes habían sido los tributarios históricos de las aguas, los campesinos indígenas agroganaderos de valles, precordillera y altiplano, resultó sintomático de la orientación modernizadora de este modelo de desarrollo regional.

Por una parte, la población andina no estuvo como un actor constituyente de los sectores provinciales que se afanaban en darle un sentido estratégico a la utilización de las existencias acuícolas de la cordillera. Por otra, tampoco hubo consideración ante el posible impacto que generaría este trasvasije tan masivo de aguas a la pampa del Tamarugal en las economías agroganaderas de las comunidades aymaras $^{47}$; en este sentido primó la preponderancia de lo moderno por sobre lo tradicional.

Es probable, de haberse dado este escenario desarrollista, que una parte de estos indígenas campesinos, los vallesteros, se hubiesen integrado plenamente a la nueva dinámica modernizadora de una agricultura a gran escala orientada al mercado. Lo anterior no hubiese sido para nada extraño, después de todo muchos de ellos ya habían experimentado esta transformación de intentar ejecutar usos hídricos asociados a proyectos agrícolas modernizadores ${ }^{48}$. Por ejemplo, los casos ya reseñados de las sociedades constituidas en la quebrada de Tarapacá en 1876 y en el valle de Camarones en $1887^{49}$, como otros intentos menores, pero con 
este mismo perfil en Camiña, Aroma, Pica, Canchones y Tiliviche ${ }^{50}$. Por el contrario, para los agroganaderos andinos del altiplano hubiese implicado un efecto negativo radical, sobre todo tomando en cuenta que sus estructuras socioeconómicas, particularmente su agricultura de subsistencia, derechamente no estaban adecuadas para una inserción tan rápida a una producción mercantilizada (Gundermann 1999).

No obstante las diferencias potenciales entre aymaras vallesteros y altiplánicos, la posibilidad de extraer gran parte de las existencias hídricas de la cordillera con el objeto de irrigar masivamente la pampa del Tamarugal finalmente hubiese operado en ambos contingentes como un factor exógeno; más aún cuando el conjunto de los proyectos en cuestión tenían por objetivo medular dar un sustrato lo suficientemente acotado a una estrategia de desarrollo regional moderna y en ningún caso entregar instrumentos específicos y en directo beneficio para las economías agroganaderas "tradicionales" y "atrasadas" de los campesinos indígenas que habitaban en el interior de la provincia de Tarapacá. En otras palabras, con la fertilización en grande de la superficie arenosa de la pampa se buscó, tal como lo indicó el Intendente Gonzalo Bulnes en 1884, modificar una "agricultura mui reducida" trabajada por "una población indígena, importante como número, si bien desprovisto de actividad i energía moral"51, o como lo reafirmó el Intendente Oyarzún Lorca en 1920 al señalar la necesidad que había de superar las actividades de “...jentes rústicas, sin ilustración, [que] cultivan sus campos en la forma rudimentaria, como lo hacían sus antepasados, forma que ha venido trasmitiéndose de generación en generación"52.

A partir de este posicionamiento, el elemento más gravitante a la hora de describir una posición precaria de la población campesina indígena ante las directrices establecidas para ocupar aguas altoandinas al amparo de un proyecto modernizador fue el de legalidad impositiva de los bienes naturales, un componente que zanjó con rapidez la propiedad fiscal de la totalidad de los recursos hídricos de la provincia. Bajo este parámetro las propuestas de desarrollo agrícola, tanto la de los agentes locales como las impulsadas por la administración estatal chilena, terminaron enajenando los intereses y conveniencias de la población aymara parlante como su experiencia cultural de larga data en lo tocante a la agricultura en el desierto. De esta manera, el aparato fiscal muy tempranamente operó con este criterio para resolver, positiva o negativamente, las solicitudes privadas de concesión de aguas, enajenando así los derechos históricos de los campesinos indígenas sobre este escaso recurso. Gonzalo Bulnes, una autoridad bastante interesada en estos asuntos, fue en buen ejemplo de este proceder. Acusando una petición para ocupar acuíferos ubicados al interior del oasis de Pica, en 1885 aclaró:

No veo qué inconveniente legal podría oponerse a la concesión del agua que se extraiga de la tierra desde que nuestra legislación permite a los particulares apropiarse del agua de los ríos i vertientes para dedicarlas al regadío de los terrenos ${ }^{53}$.

Si lo afirmado por Bulnes a fines del mes de agosto de 1885 dejaba en claro los fundamentos legales en los que se sustentaría la administración estatal chilena para actuar en esta materia, lo que eliminaba en principio cualquier atisbo de reclamación basada en derechos consuetudinarios amparados por la legislación peruana, las acciones emprendidas por el gobierno en 1890 -como la formación de una Comisión Fiscal encargada de evaluar todos los estudios hidrográficos y geológicos que llevaran a cabo empresarios privados y la ya referenciada designación de Juan Schulze para que ejecutara estudios de factibilidad tendientes a sacar agua para regar la pampa salitrera y abastecer Iquique ${ }^{54}$ - terminaron por reafirmar la total asimetría y desventaja de los campesinos aymaras con relación a los derechos que podía ejercer el propio Estado chileno como los restantes ciudadanos en pos de ocupar recursos hídricos con fines económicos.

\section{Conclusiones}

Entre las décadas de 1880 y 1930 importantes sectores de la vida pública de la provincia de Tarapacá se manifestaron a favor de usar los recursos hídricos para irrigar la pampa del Tamarugal, una inquietud generalizada que dio paso a una corriente de opinión pública que terminó cuestionando los énfasis estatales por restringir la economía tarapaqueña a la sola explotación salitrera. El an- 
helo por trasvasijar grandes volúmenes de agua e irrigar masivamente se afianzó rápidamente en distintos grupos gremiales de Iquique que se abocaron a formular proyectos o promover propuestas de terceros buscando demostrar la viabilidad de esta idea, sobre todo de una agricultura moderna orientada al mercado y a la exportación.

En este escenario de gran dinamismo la posición que tomaron algunos intendentes resultaron de suyo interesante, sobre todo porque, a pesar de que eran los representantes del Ejecutivo en la provincia, fueron capaces de asumir ciertas posturas regionalistas, demostrando de paso que tenían una visión macro de las potencialidades económicas de Tarapacá.

No obstante lo anterior, desde la perspectiva de los intereses y requerimientos de los campesinos aymaras finalmente no hubo mayor distancia entre el accionar fiscal propenso a utilizar los acuíferos para abastecer los centros urbanos y satisfacer los requerimientos de la industria salitrera y las intenciones de los partidarios, en su gran mayoría residentes iquiqueños, de incentivar un sector agrícola mercantil a gran escala como parte de un proyecto de desarrollo regional no rentista. En este sentido, la actividad agrícola de las poblaciones indígenas, vista como tradicional y no moderna, quedó marginada de los lineamientos centrales que encauzaron el debate y las propuestas agrarias.

Agradecimientos: Este artículo forma parte del proyecto Dipuv 01/2000 “Economía y Sociedad en Tarapacá: Comunidad regional, Estado nacional y la problemática del desarrollo, 1880-1940”, Dirección de Investigación y Postgrado, Universidad de Valparaíso.

\section{Referencias Citadas}

AIT

1884 Archivo Nacional de Chile. Fondo Intendencia de Tarapacá. Volúmenes 67, 68.

1885 Volúmenes 46, 76.

1886 Volumen 15.

1888 Volumen 151.

1889 Volumen 157

1890 Volúmenes 181, 536.

1894 Volumen 177.

1903 Volumen 492.

AITRI

1909 Archivo Regional de Iquique. Fondo Intendencia de

Tarapacá. Libro Arrendamientos de Terrenos Fiscales 1909.

Volumen 2.

1909 Volumen 23, Libro Solicitudes 1909.

1920 Volumen 6, Libro Oficios a los Ministerios 1930.

1922 Volumen 32, Libro Copiador de Oficios a los Ministerios 1922.

1923 Volumen 10, Libro Ministerio Industria y Obras Públicas 1923 .

1928 Volumen 1, Libro Ministerio de Obras Públicas y Fomento 1920-1928.

1932 Volumen 11, Libro Memorias 1932.

Alfaro, C.

1936 Reseña histórica de la Provincia de Tarapacá. Imprenta AMI

Caras y Caretas, Iquique.

1887 Archivo Nacional de Chile. Fondo Ministerio del Interior.

Volumen 1406.

AMIOP

1894 Archivo Nacional de Chile. Fondo Ministerio de Obras Públicas.

Volumen 694.
1915 Volumen 2619.

1919 Volumen 2815.

ANI

1876 Archivo Nacional de Chile. Fondo Notarial de Iquique. Volumen 18, Registro 62, Documento 22.

ANT

1876 Archivo Nacional de Chile. Fondo Notarial de Tarapacá.

Volumen 14, Protocolo 1876, Minuta 79.

Bermúdez, $\mathrm{O}$.

1963 La Historia del Salitre desde sus Orígenes hasta la Guerra del Pacífico. Ediciones de la Universidad de Chile, Santiago.

s/f La "agricultura sin riego" en la zona de Canchones (Tarapacá, Norte de Chile). Manuscrito inédito.

Billinghurst, G.

1886 Estudio sobre la Geografía de Tarapacá. Santiago. 1887 El abastecimiento de Agua Potable del Puerto de Iquique. Imprenta Española, Iquique.

1893 La Irrigación de Tarapacá. Imprenta Ercilla, Santiago. BOIFMIT

1936 Boletín Oficial del Instituto de Fomento Minero e Industrial de Tarapacá, Año II (2), 25 de octubre, Iquique.

Carevic, A.

1989 La Pampa del Tamarugal: una visión arqueológica y etnohistórica de su utilización. Documentos de Investigación 10:1-16, Centro de Estudios del Desierto, Universidad Arturo Prat, Iquique.

Castro, L.

1995 Tarapacá 1880-1936: Debates, reflexiones, propuestas y proyectos en torno al problema del desarrollo. Temas Regionales, Año 2 (1):32-66.

1998a Cuando el susurro del agua se acalló en el desierto: La expropiación de las aguas del valle de Quisma, el abastecimiento fiscal a Iquique y el debate sobre el pro- 
blema del desarrollo económico regional, Tarapacá 19121937. Tesis Magíster en Historia, Universidad de Santiago.

1998b Las otras luchas sociales en el Tarapacá salitrero. La defensa de los quismeños del agua de Chintaguay. En A 90 años de los Sucesos de la Escuela Santa María de Iquique, pp. 45-78, Coedición Dibam, Lom, Centro de Investigaciones Barros Arana, Universidad Arturo Prat, Iquique.

2002 La circulación arterial de la riqueza: Estrategias de desarrollo regional e integración económica con Bolivia y el noroeste argentino, Tarapacá 1864-1936. Diálogo Andino 19:111-131.

2003 Regionalismo y Desarrollo Regional: Debate público, proyectos económicos y actores locales (Tarapacá 18801930). Manuscrito inédito.

Contreras, C. y M. Cueto

1999 Historia del Perú Contemporáneo. Red para el Desarrollo de las Ciencias Sociales, Lima.

Couyoumdjian, R. y H. Larraín

1975 El plano de la Quebrada de Tarapacá de don Antonio O'Brien. Su valor geográfico y socio antropológico. Norte Grande, vol.1 (3-4):329-362.

El Nacional

1900 Iquique, 22 de abril

El Regional

$1936 \mathrm{~N}^{\mathrm{o}}$ 2, Iquique, 19 de diciembre

Felsch, J.

1920 El agua en la pendiente occidental de la Cordillera Real entre la Quebrada de Huatacondo y la Quebrada de Tarapacá. Publicaciones del Servicio Geológico 5, Ministerio de Obras Públicas, Santiago.

Guerrero, V.

1991 Recursos hídricos y conflictos sociales en Tarapacá. Cuadernos de Investigación Social 31:1-38, CREAR, Iquique.

Gundermann, $\mathrm{H}$.

1997 Etnicidad, identidad étnica y ciudadanía en los países andinos y el Norte de Chile. Los términos de la discusión y algunas hipótesis de investigación. Estudios Atacameños 13:9-26.

1998 Comunidad aymara, identidades colectivas y estados nacionales en los albores del siglo XX. En A 90 años de los sucesos de la Escuela Santa María de Iquique, pp. 45-78, Coedición Dibam, Lom, Centro de Investigaciones Barros Arana, Universidad Arturo Prat, Iquique.

1999 Sociedad aymara y cambios sociales en la segunda mitad del siglo XIX. Ponencia, Terceras Jornadas de Historia Andina del Norte de Chile, Universidad de Valparaíso.
Hidalgo, J.

1985 Proyectos coloniales inéditos de riego en el desierto. Azapa (cabildo de Arica, 1619), pampa Iluga (O’Brien, 1765) y Tarapacá (Mendizábal, 1807). Chungara 14:183222.

Keller, C.

1946 El Departamento de Arica. Empresa Editora Zig-Zag, Santiago.

La Defensa de Tarapacá

1934 Iquique, 17 de noviembre

La Patria

1894 Iquique, 7 de diciembre

Larraín, H.

1974 Antecedentes históricos para un estudio de la reutilización de los suelos agrícolas en la Pampa del Tamarugal. Norte Grande, vol.1(1):9-22.

Las Cuestiones Sociales

$1922 \mathrm{~N}^{\circ} 46$, Iquique, 12 de enero; $\mathrm{N}^{\circ} 47$, Iquique, 19 de enero. $1926 \mathrm{~N}^{\circ} 285$, Iquique, 12 de diciembre

López Loayza, F.

1913 La provincia de Tarapacá (Alrededor de su industria y de Iquique, su principal puerto). Editor Edw. E. Muecke, Iquique.

Martínez, V.

1893 Proyecto de Abastecimiento de Agua Potable para el Puerto de Iquique y otras Poblaciones del Departamento de Tarapacá. Santiago.

Memoria del Administrador de la Aduana y Tesorería Unida de Iquique al Superintendente de Aduanas

1912 En Fernando López Loayza, La Provincia de Tarapacá (Alrededor de su Industria y de Iquique, su Principal Puerto). Editor Edw. E. Muecke, Iquique.

Podestá, J.

2004 La invención de Tarapacá. Estado y Desarrollo Regional en Chile. Ediciones Campvs, Universidad Arturo Prat, Iquique.

Ríos, M.

1992 Política y propuestas de desarrollo en Arica desde 1930 a 1973. Cuadernos de Investigación Social 32:1-52, CREAR, Iquique.

Salinas, M.

1903 Proyecto de irrigación de la Pampa del Tamarugal presentado al Congreso Nacional, Santiago, 29 de mayo de 1903. En Provincia de Tarapacá, editado por Francisco Riso-Patrón, pp. 34-38, Santiago.

Van Kessel, J.

1985 La lucha por el agua en Tarapacá: la visión andina. Chungara 14:141-155.

1992 Holocausto al Progreso. Los Aymaras de Tarapacá. HISBOL, La Paz.

\section{Notas}

1 Las paradas eran grandes ollas de fierro donde se calentaba a leña agua mezclada con soluciones químicas para ejecutar la lixiviación, o sea, la separación del mineral salino del material estéril.

2 Según Oscar Bermúdez (1963:133, nota 1), el químico italiano Antonio Raimondi llegó a Tarapacá a fines de 1853 mandado por el gobierno peruano para analizar vetas mineras de boratos de soda y cal que se habían descubierto por aquella época. En esta categoría de asesor minero, estuvo ligado a esta provincia hasta la década de 1870 . Este científico llegó muy joven al Perú, en 1850, desarrollando una notable carrera académica en la Universidad de San 
Marcos en donde dictó los cursos de Historia Natural y Química Analítica; además, realizó notables estudios de geografía, botánica y mineralogía. Todo lo anterior lo llevó a convertirse en el naturalista más importante del siglo XIX en ese país (Contreras y Cueto 1999:104).

3 ANI, vol. 18 , reg. 62 , doc. 22 , Iquique $21 / 6 / 1876$, fol. $27 \mathrm{v}$ 28; ANT, vol. 14, protocolo 1876, minuta 79, San Lorenzo de Tarapacá 20/6/1876, fol. 73-77.

El registro de la sociedad en las Notarías de Iquique y Tarapacá presenta leves diferencias en el número de socios. En la primera inscripción aparecen 33 participantes y en la segunda 49. No tengo mayores antecedentes de esta anomalía, salvo la presunción que en la Notaría de Tarapacá, por estar ubicada en el valle mismo, pudieron firmar aquellos que les fue imposible bajar a Iquique.

4 AIT, vol. 65, Informe del Intendente de Tarapacá sobre la solicitud de Claudio Mackenna para ocupar diferentes aguadas de la provincia, Iquique 20/12/1884, sin fol.

5 AIT, vol. 68, Informe del Intendente de Tarapacá al Ministro de Obras Públicas respecto a la petición de Claudio Mackenna para utilizar diversas aguadas con el objeto de regar la pampa del Tamarugal, Iquique junio 1884, sin fol.

6 AIT, vol. 76, Informe sobre la solicitud de Francisco $S$. Olivan y Cía. para obtener una concesión de aguas subterráneas, Iquique 29/8/1885, fol. 12-12v.

7 Ibíd.

8 AIT, vol. 151, Informe de la Comisión de Agua Potable sobre solicitudes diversas, Iquique 23/8/1888, fol. 102.

9 AIT, vol. 15, Solicitud de concesión de agua en Pica, Iquique 1886 , sin fol.

10 AIT, vol. 151, Informe de la Comisión de Agua Potable sobre solicitudes diversas, Iquique 23/8/1888, fol. 102.

11 AMI, vol. 1406, Memoria del Gobernador de Pisagua, Pisagua 13/4/1887, fol. 240.

12 AIT, vol. 157, Solicitudes concesión de agua, Iquique 15/2/1889, fol. 24-25.

13 Ibíd.

14 AIT, vol. 181, Informe del Intendente de Tarapacá sobre solicitud de aguas de la laguna del Huasco, Iquique 22/2/ 1890, fol. 15-16.

15 AIT, vol. 536, Oficio del Ministro de Industria y Obras Públicas al Intendente de Tarapacá dando cuenta del decreto $N^{o} 253$, Santiago 22/1/1890, sin fol.

16 La Patria, Iquique 7/12/1894.

17 AIT, vol. 492, Copias de los decretos de concesión de las aguadas de Guatacondo a Manuel Risueño, Santiago 28/3/1903, sin fol.

18 AMIOP, vol. 2619, Documento confidencial: Agua Potable Iquique. Minuta explicativa de la situación producida en las obras de captación, Iquique 1915, sin fol.; Las Cuestiones Sociales, N 47, Iquique 19/1/1922, p. 2. Ibíd.

20 AITRI, vol. 2, Arrendamiento de Terrenos Fiscales 1909, Concesión aguas de Lirima, Santiago 28/12/1908, sin fol.

21 AMIOP, vol. 2619, Memorando sobre el agua potable de Iquique, Santiago 1915, sin fol.

22 Entre los que firmaron este documento dirigido al Intendente de Tarapacá y a la comunidad iquiqueña figuraban: Ernesto Escalante, Luis Cancino, Camilo Ayarza, Roberto León Viel, Carlos Gahona, Adolfo Ramírez, Pedro
Bello, Luis Castro, Santiago Silva, Pablo Di Caro, Teodoro Blanche, etc.

23 AITRI, vol. 23, Solicitudes 1909, Manifiesto de vecinos de Iquique al Intendente de Tarapacá apoyando el uso de las aguas de Chuncara y Lirima, Iquique 2/9/1909, sin fol.

24 Las Cuestiones Sociales, No 47, Iquique 19/1/1922, p. 2.

25 AMIOP, vol. 2619, Memorando sobre el agua potable de Iquique, Santiago 1915, sin fol.

26 AMIOP, vol. 2815, Oficio del Director de Obras Públicas al Ministro de Obras Públicas, Santiago 25/4/1919, sin fol.

27 AITRI, vol. 32, Copiador de Oficios a los Ministerios 1922, Solicitud mercedes de agua, Iquique 22/5/1922, fol. 402.

28 AITRI, vol. 10, Ministerio de Industria y Obras Públicas 1923, Concesión aguas de Aroma, Santiago 15/12/1923, sin fol.

29 Ibíd

30 AIT, vol. 68, Informe del Intendente de Tarapacá al Ministro de Obras Públicas respecto a la petición de Claudio Mackenna para utilizar diversas aguadas con el objeto de regar la pampa del Tamarugal, Iquique junio 1884, sin fol.

31 AIT, vol. 177, Informe del Ingeniero de la Provincia al Intendente de Tarapacá sobre la irrigación de la pampa del Tamarugal, Iquique 9/7/1894, sin fol.

32 AMIOP, vol. 694, Oficio del Intendente de Tarapacá al Ministro de Obras Públicas, Iquique 6/9/1894, sin fol.

33 Para dimensionar el nivel de participación del periodismo iquiqueño en este debate, sólo es necesario recordar cómo su labor, mediante los gravitantes editoriales y reiterados artículos, fue primordial para masificar la discusión y transformarla en una cuestión pública.

34 La participación de la Iglesia Católica tarapaqueña en el debate sobre el desarrollo regional y la apuesta por disminuir la preponderancia de la renta salitrera en la economía provincial se canalizó a través del semanario Las Cuestiones Sociales, publicación oficial de difusión de sus ideas, que acotó en sus ediciones una buena parte a opinar argumentadamente sobre estas materias denotando una decidida postura regionalista.

35 El quehacer empresarial de estos personajes ligado a proyectos agrícolas se puede revisar en: Bermúdez (s/f:411423) y Carevic (1989:5-20).

36 El Nacional, Iquique 22/4/1900.

37 Memoria del Administrador de la Aduana y Tesorería Unida de Iquique al Superintendente de Aduanas. Iquique 1912. En: López Loayza (1913:56).

38 Las Cuestiones Sociales, No 46, Iquique 12/1/1922, p. 2

39 Las Cuestiones Sociales, № 285, Iquique 9/12/1926, p. 4.

$40 \quad$ La Defensa de Tarapacá, Iquique 17/11/1934, p. 10.

41 El fracaso de los embalses de Pachica y Caritaya como instrumentos de desarrollo económico no sólo se acotó al término de un ciclo (1880-1930), sino que adicionalmente se vinculó con las dificultades económicas que vivió Tarapacá en el lapso de las décadas de 1940 a 1970. Este último período es tratado, para el caso de la provincia de Arica, por Ríos (1992) y para la región de Tarapacá por Podestá (2004).

42 La opinión del obispo Labbé no fue un asunto formal de alguien con autoridad moral para inmiscuirse en los problemas económicos locales, sino una voz autorizada y competente. Como ya lo indiqué, la Iglesia Católica, a través del semanario Las Cuestiones Sociales, se constituyó en 
un actor de primer orden en la promoción de un proyecto de desarrollo regional; pero, además, Labbé fue un decidido impulsor a través de la creación del fundo El Carmelo, cercano al pueblo de La Tirana, de una de las iniciativas agrícolas más interesante de aquella época; por tanto, sabía perfectamente cuánto preocupaba el tema del manejo de los recursos hídricos.

43 AITRI, vol. 1, Ministerio de Obras Públicas y Fomento 1920-1928, Aprobación de planos del proyecto de riego del valle de Camarones, Santiago 8/6/1928, sin fol.

44 Mayores antecedentes sobre esta iniciativa ferroviaria en: Castro (2002).

45 AITRI, vol. 11, Memorias 1932, Informe sobre los embalse de Caritaya y Pachica, Caritaya 5/1/1932, sin fol.

46 El Regional, $\mathrm{N}^{\circ}$ 2, Iquique 19/12/1936, p. 5.

47 Para el concepto "comunidad aymara" seguimos el planteamiento de Hans Gundermann $(1998: 153-181$; 1999) que señala la existencia de ésta sólo en términos sociológicos y no histórico-cultural, denotando con ello los cambios estructurales en la sociedad andina de fines del siglo XIX y comienzos del XX.

48 Hans Gundermann $(1997: 11 ; 1999)$ describe esta experiencia, pero en un marco de comprensión mayor, como la confi- guración de un nexo estructural de los campesinos aymaras de valles con la economía capitalista de enclave de la minería salitrera, vínculo con claras implicaciones de integración a los mercados subregionales.

49 ANI, vol.18, reg. 62, doc. 22, Iquique 21/6/1876, fol. 27r28; ANT, vol. 14, protocolo 1876, minuta 79, San Lorenzo de Tarapacá 20/6/1876, fol. 73-77; AMI, vol. 1406, Memoria del Gobernador de Pisagua, Pisagua 13/4/1887, fol. 240.

50 AMI, vol. 1406, Memoria del Gobernador de Pisagua, Pisagua 13/4/1887, fol. 240.

51 AIT, vol. 67, Descripción de Tarapacá, Iquique 5/6/1884, $\sin$ fol.

52 AITRI, vol. 6, Oficios a los Ministerios 1930, Informe del Intendente de Tarapacá al Ministro del Interior sobre los problemas agrícolas de Tarapacá, Iquique 12/11/1920, $\sin$ fol.

53 AIT, vol. 46, Solicitudes de aguas por parte de Francisco S. Olivan y Cía., Iquique 29/8/1885, fol. 12r.

54 AIT, vol. 181, Informe del Intendente de Tarapacá Ramón Yávar al Ministro del Interior, Iquique 22/2/1890, sin fol.; AIT, vol. 536, Oficio del Ministerio de Obras Públicas al Intendente de Tarapacá, Santiago 22/1/1890, sin fol. 